\title{
A TIME-DOMAIN ANALYSIS OF WAVE FORCE ON SMALL-SCALE CYLINDERS OF OFFSHORE STRUCTURES
}

\section{Bao-Lei Geng}

State Key Laboratory of Coastal and Offshore Engineering, Dalian University of Technology, Dalian, P.R. China.Key Laboratory of Engineering Sediment of Ministry of Communications, Tianjin Research Institute for Water

Transportation Engineering, Tianjin, P.R.China, stonegeng@163.com

\section{Bin Teng}

State Key Laboratory of Coastal and Offshore Engineering, Dalian University of Technology, Dalian, P.R. China

De-Zhi Ning

State Key Laboratory of Coastal and Offshore Engineering, Dalian University of Technology, Dalian, P.R. China

Follow this and additional works at: https://jmstt.ntou.edu.tw/journal

Part of the Civil and Environmental Engineering Commons

\section{Recommended Citation}

Geng, Bao-Lei; Teng, Bin; and Ning, De-Zhi (2010) "A TIME-DOMAIN ANALYSIS OF WAVE FORCE ON SMALL-SCALE CYLINDERS OF OFFSHORE STRUCTURES," Journal of Marine Science and Technology. Vol. 18: Iss. 6, Article 12. DOI: $10.51400 / 2709-6998.1946$

Available at: https://jmstt.ntou.edu.tw/journal/vol18/iss6/12

This Research Article is brought to you for free and open access by Journal of Marine Science and Technology. It has been accepted for inclusion in Journal of Marine Science and Technology by an authorized editor of Journal of Marine Science and Technology. 


\section{A TIME-DOMAIN ANALYSIS OF WAVE FORCE ON SMALL-SCALE CYLINDERS OF OFFSHORE STRUCTURES}

\section{Acknowledgements}

The authors gratefully acknowledge the financial support provided by the National Science and Technology Major Project of China (Grant No. 2008ZX05026-02-02); Natural Science Foundation of China (Grant No. 10772040, 50709005) and Central Commonweal Research Institute Basic R\&D Special Foundation of TIWTE (Grant No. TKS090201). 


\title{
A TIME-DOMAIN ANALYSIS OF WAVE FORCE ON SMALL-SCALE CYLINDERS OF OFFSHORE STRUCTURES
}

\author{
Bao-Lei Geng***, Bin Teng*, and De-Zhi Ning*
}

Key words: small-scale cylinders, wave force, time domain analysis, offshore structures.

\begin{abstract}
To get the accurate wave loads on the small-scale cylinders on the platforms, not only the incident wave but also the diffraction and radiation wave should be considered. By taking the wave diffraction from a fixed large-scale cylinder as an example, wave loads on small-diameter cylinders in the diffraction wave field are calculated by Morison formula in this paper. A time-domain numerical model on wave diffraction from large-scale structures is developed with a higher-order boundary element method (HOBEM). Then the velocity and acceleration of any water particle in the fluid domain can be produced from the solved diffraction potential. Results show that the incident wave force is even equal to diffraction wave force when the diameter of the large-scale cylinder is 8 times as great as the small-scale one; And the total force is different with the locations of the small-scale cylinder. The maximum total force is 1.57 times as great as only the incident wave force. Therefore, the wave force on the stake produced by the diffraction waves due to the existence of upper structures should be considered in the practical engineering.
\end{abstract}

\section{INTRODUCTION}

As typical deep sea platforms, a lot of Spar platforms have been built in the Gulf of Mexico and the North Sea by large petroleum corporations. This platform mode mostly involves complicated structure composed of large-scale tanks and smallscale members. In the engineering design, diffraction theory and Morison equation [15] were adopted separately to calculate the wave loads on the structure based on different ratio of the characteristic length (e.g., diameter $D$ of column structure)

Paper submitted 10/07/09; revised 11/05/09; accepted 11/09/09. Author for correspondence: Bao-Lei Geng (e-mail: stonegeng@163.com).

*State Key Laboratory of Coastal and Offshore Engineering, Dalian University of Technology, Dalian, P.R. China.

**Key Laboratory of Engineering Sediment of Ministry of Communications, Tianjin Research Institute for Water Transportation Engineering, Tianjin, P.R. China. to wavelength $L$ : when $D / L>0.2$, the diffraction theory was adopted; when $D / L<0.2$, the Morison equation is used. A quantitative chart for differentiating small and large structure has been given in Chakrabarti [3]. Chakrabarti et al. [4] also analyzed the response of a TPS (truss pontoon semi-submersible) by applying Morison equation and linear diffraction theory, and the analytical heave and pitch motion results were compared with the model test results. His study suggests that the linear part of the Morison equation can be applied to analyze the platform as a simplified method.

Morison equation has two components: velocity force and inertia force, which are related to velocity and acceleration of the water particle caused by wave, respectively. The influence on flow field caused by small-scale structure was omitted. The velocity and acceleration are calculated from incident wave in simple wave field. For the small-scale structure near largescale structure (just like truss members of a Truss Spar and SAL (single anchor leg) nearby a FPSO, see Fig. 1), the influence on flow field generated due to the existence of largescale structure can not be omitted. But some people still use incident wave to calculate the wave force on small-scale structures, and this will result in calculation errors. For example, Kim et al. [11] calculated the wave and current load on the truss of the Amoco Marlin truss Spar by Morison equation, but he did not specify whether the wave scattering effect from hard tank was taken into account. Zhang et al. [17] performed calculation on the truss of a Cell-Truss Spar platform and he

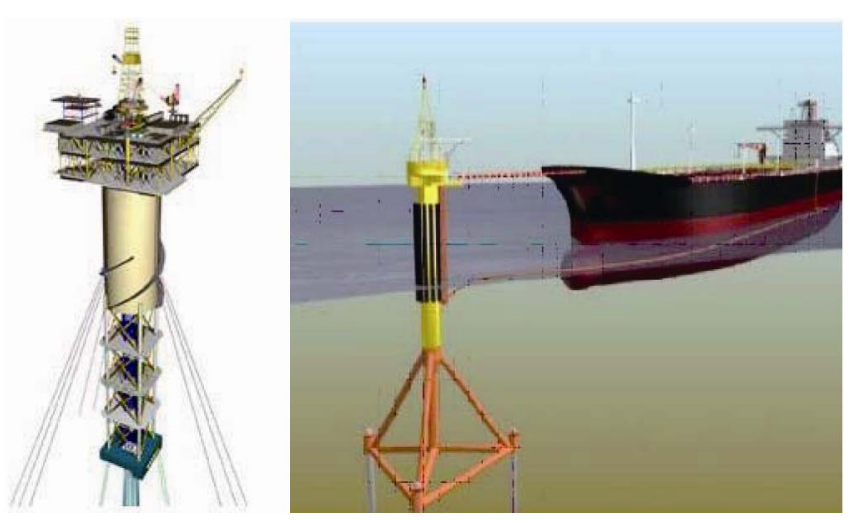

Fig. 1. Small-scale structures near large-scale structures. 
used linearized Morison equation only. Kurian et al. [12] used Morison equation to compute the normal wave forces on the hard tank and truss members and used force coefficients to compensate for scattering effect. Chen et al. [6] provided a method to predict the load and calculate the fatigue strength of tubular joints, and he also did not show whether the wave scattering effect from large-scale body was taken into account in Morison equation. Luo et al. [14] analyzed the fatigue of truss Spar's critical connections in time domain using the same method as Kurian, therefore, the same problem existed.

In the present study, by taking wave diffraction from a complicated structure, composed of large-scale cylinders and small-scale members, as an example, the influence of diffraction field on the stress of small-scale members was analyzed. In the analysis: a boundary integral equation for the diffraction potential due to large-scale structure was established in the computational domain according to the second Green's identity, then a high-order boundary element method $[1,5,16]$ was employed to calculate the wave diffraction potential in time domain. By making use of known and solved velocity potential and its' normal derivative on the whole boundaries, the velocity and acceleration of water particles in the domain can be obtained through the integral equation. Morison formula was used to solve the incident and diffraction wave force on small-scale members. Numerical results show that under certain wave condition, the influence of wave diffraction generated due to the existence of large-scale structure on small-scale members can not be omitted.

\section{BASIC CALCULATION PRINCIPLE}

Considering a problem of structure diffraction induced by incident waves propagating along the $x$-direction at water depth $d$, a right-hand coordinate system $O x y z$ is established with the origin $O$ on the static water surface and the axis $O z$ is measured vertically upward, as shown in Fig. 2.

For wave diffraction from large-scale object, the diffraction theory based on potential-flow theory can be adopted for calculation. Thus the wave field includes incident waves and diffraction waves generated due to the existence of large-scale objects, can be obtained. Morison formula can be used to solve the interaction between waves and small-scale objects.

\section{Calculation Method for Wave Diffraction from Large-Scale Structure in Time Domain}

For the interaction between linear incident waves and works, velocity potential $\Phi$ and wave-elevation $\eta$ can be divided into a known incident component and a diffraction component as follows

$$
\begin{gathered}
\Phi=\Phi_{\mathrm{i}}+\Phi_{\mathrm{s}} \\
\eta=\eta_{\mathrm{i}}+\eta_{\mathrm{s}}
\end{gathered}
$$

where subscripts $i$ and s represent the incident and diffraction

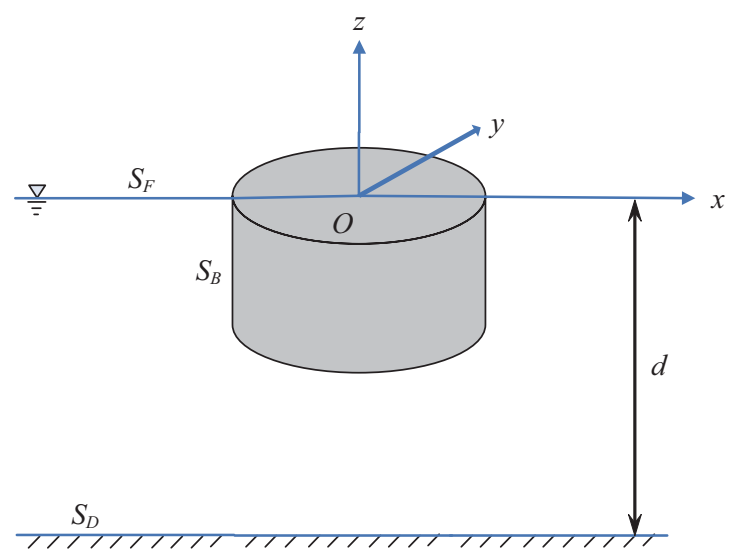

Fig. 2. Definition Sketch.

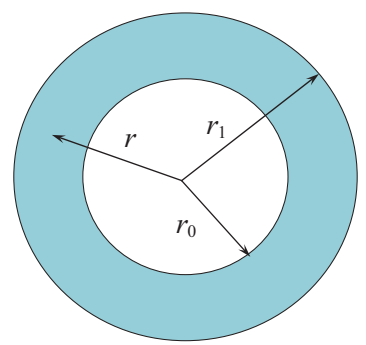

Fig. 3. Sketch of damping layer.

components, respectively.

Under the assumptions that the fluid motion is irrotational and the fluid is incompressible and invisid, the diffraction potential satisfies Laplace equation in the fluid domain

$$
\nabla^{2} \Phi_{\mathrm{s}}=0
$$

and the following solid boundary conditions

$$
\begin{array}{cc}
\frac{\partial \Phi_{\mathrm{s}}}{\partial z}=0 & (z=-d) \\
\frac{\partial \Phi_{\mathrm{s}}}{\partial n}=-\frac{\partial \Phi_{\mathrm{i}}}{\partial n} & \left(\text { on } S_{B}\right)
\end{array}
$$

Here $n$ denotes distance in the direction of the unit normal vector $\mathbf{n}=\left(n_{x}, n_{y}, n_{z}\right)$ directed outward from the fluid region.

Both kinematic and dynamic boundary conditions are satisfied on the free water surface. To make long time simulation within limited computational domain, the reflection waves is dissipated by using a damping layer in outer field as shown in Fig. 3 [7]. To realize it, a damping term is added into the free surface boundary conditions, as the following:

$$
\frac{\partial \eta_{\mathrm{s}}}{\partial t}=\frac{\partial \Phi_{\mathrm{s}}}{\partial z}-v(r) \eta_{\mathrm{s}}
$$




$$
\frac{\partial \Phi_{\mathrm{s}}}{\partial t}=-g \eta_{\mathrm{s}}-v(r) \Phi_{\mathrm{s}}
$$

where

$$
v(r)= \begin{cases}\kappa \omega\left(\frac{r-r_{0}}{\lambda}\right)^{2} & \left(r_{0} \leq r \leq r_{1}=r_{0}+\beta \lambda\right) \\ 0 & \left(r<r_{0}\right)\end{cases}
$$

$\kappa$ is the damping coefficient; $\beta$ is the width coefficient of the damping layer; $\lambda$ is the characteristic wave length. In order to improve the efficiency in the damping area, a proper coefficient must be adopted so that diffracted waves can be absorbed completely. Here 1.0 is taken for both $\kappa$ and $\beta$ in calculation [2].

By using the second Green's identity, the following boundary integral equation about diffraction potential $\Phi_{\mathrm{s}}$ can be obtained:

$$
\alpha \Phi_{\mathrm{s}}\left(\xi_{0}\right)=\iint_{S}\left[\frac{\partial G\left(\xi, \xi_{0}\right)}{\partial n} \Phi_{\mathrm{s}}(\xi)-\frac{\partial \Phi_{\mathrm{s}}(\xi)}{\partial n} G\left(\xi, \xi_{0}\right)\right] d s
$$

where $\xi_{0}=\left(x_{0}, y_{0}, z_{0}\right)$ represents the source point; $\xi=(x, y, z)$ represents the field point. $\alpha$ is a solid angle coefficient and satisfies the following forms:

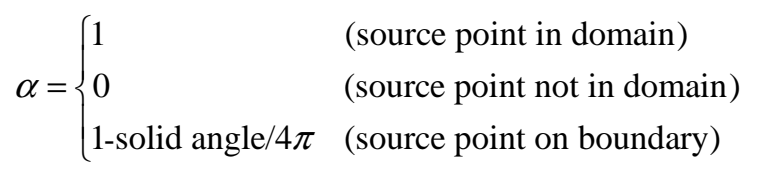

Boundary $S$ includes submerged mean object surface $S_{B}$ and limited static water surface $S_{F}$ from an object to the external boundary of the damping layer. Rankine source and its image of sea bottom were chosen as Green function.

$$
\begin{gathered}
G\left(\xi, \xi_{0}\right)=-\frac{1}{4 \pi}\left(\frac{1}{R}+\frac{1}{R_{1}}\right) \\
R^{2}=\left(x-x_{0}\right)^{2}+\left(y-y_{0}\right)^{2}+\left(z-z_{0}\right)^{2} \\
R_{1}^{2}=\left(x-x_{0}\right)^{2}+\left(y-y_{0}\right)^{2}+\left(z+z_{0}+2 d\right)^{2}
\end{gathered}
$$

The boundary surface is discretized into a set of higherorder boundary elements, which are composed of eight-node quadrilateral elements and six-node triangular elements. They can be transformed to isoparametric elements in local coordinates. Thus the velocity potential and the geometry coordinates in each element can be interpolated by the values of various nodes and shape functions. In the calculation, the current normal derivative of the velocity potential on object surface and the velocity potential on free surface are known. The velocity potential on object surface and the normal derivative on free surface are calculated by the integral equation (8). Then the wave elevation and the velocity potential on the free water surface at the next moment are calculated with numerical integration method according to the free water surface conditions (6) and (7), and the normal derivative at object surface at the next moment can be obtained according to object-surface condition (5). The calculation was repeated till the calculation period ended $[9,10]$.

\section{Method for Calculating Wave Force on Small-Scale Members}

The wave force on a small-scale member was calculated with Morison formula which is a semi-theoretical and empirical formula, including two terms: inertia force and velocity force $[8,15]$.

$$
F=f_{\mathrm{i}}+f_{\mathrm{d}}
$$

where $f_{\mathrm{i}}$ represents the inertia force whose form is the same as the solution to non-viscous fluid based on wave theory; $f_{\mathrm{d}}$ is the velocity force whose form is similar to the resistance produced on an object in steady flow. The formulas are as follows:

$$
\begin{gathered}
f_{\mathrm{i}}=C_{\mathrm{m}} \rho \frac{\pi D^{2}}{4} \frac{\partial u}{\partial t} \\
f_{\mathrm{d}}=C_{\mathrm{d}} \frac{\rho}{2} D u|u|
\end{gathered}
$$

where $C_{\mathrm{m}}$ and $C_{\mathrm{d}}$ represents the coefficients of inertia force and velocity force, respectively; $\rho$ is the water density; $D$ is member diameter; $u$ is the velocity of water particle; $\frac{\partial u}{\partial t}$ represents the velocity of water particle. Here $C_{\mathrm{m}}$ and $C_{\mathrm{d}}$ are chosen to be 2.0 and 1.2 respectively for the results in this paper according to China standard (JTJ 213-98).

The velocity $u$ and acceleration $\frac{\partial u}{\partial t}$ of water particle are composed of incident wave and diffraction wave, respectively. The part induced by incident wave can be easily obtained through the analytical expression; the others induced by diffraction wave are solved by wave diffraction theory. Once an object's wave diffraction effect is obtained, the water-particle velocity induced by diffraction potential in $x$-direction at an arbitrary point inner the fluid domain can be solved with the following formula:

$$
\begin{aligned}
u_{\mathrm{s}, x} & =\frac{\partial \Phi_{\mathrm{s}}\left(\xi_{0}\right)}{\partial x} \\
& =\iint_{S}\left[\frac{\partial^{2} G\left(\xi, \xi_{0}\right)}{\partial n \partial x} \Phi_{\mathrm{s}}(\xi)-\frac{\partial \Phi_{\mathrm{s}}(\xi)}{\partial n} \frac{\partial G\left(\xi, \xi_{0}\right)}{\partial x}\right] d s
\end{aligned}
$$




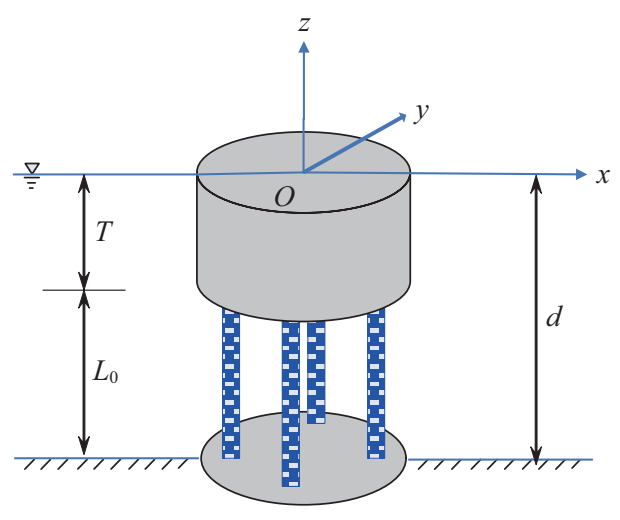

Fig. 4. Sketch of structure.

Then, the corresponding water-particle acceleration can be solved:

$$
a_{\mathrm{s}, x}=\frac{\partial u_{\mathrm{s}, x}}{\partial t}=\frac{u_{\mathrm{s}, x}^{t+1}-u_{\mathrm{s}, x}^{t}}{\Delta t}
$$

\section{NUMERICAL RESULTS AND DISCUSSIONS}

As an example, a linear wave interaction with a fixed composite structure is considered as shown in Fig. 4. The structure is composed of an upper large-scale cylinder with radius $a$ and draft $T / a=2.0$ and several small-scale piles with radius $b / a=$ 0.25 and length $L_{0} / a=2.0$. The water depth is $d / a=4.0$. In order to investigate the influence of upper structure on the wave force on small-scale pile, it is presumed that one of the piles is right beneath the cylinder.

The analytical incident wave potential and wave height can be expressed as follows:

$$
\begin{gathered}
\Phi_{\mathrm{i}}=\frac{g A}{\omega} \frac{\cosh (k(z+d))}{\cosh k d} \sin (k x-\omega t) \\
\eta_{\mathrm{i}}=A \cos (k x-\omega t)
\end{gathered}
$$

Then the corresponding horizontal velocity and the acceleration can be obtained

$$
\begin{aligned}
& u_{\mathrm{i}, x}=\frac{\partial \Phi_{\mathrm{i}}}{\partial x}=\frac{k g A}{\omega} \frac{\cosh k(z+d)}{\cosh k d} \cos (k x-\omega t) \\
& a_{\mathrm{i}, x}=\frac{\partial u_{\mathrm{i}, x}}{\partial t}=k g A \frac{\cosh k(z+d)}{\cosh k d} \sin (k x-\omega t)
\end{aligned}
$$

In this case, the time step is one-fiftieth of the wave period. The computational mesh contains 160 (16 (circumferential) $\times$
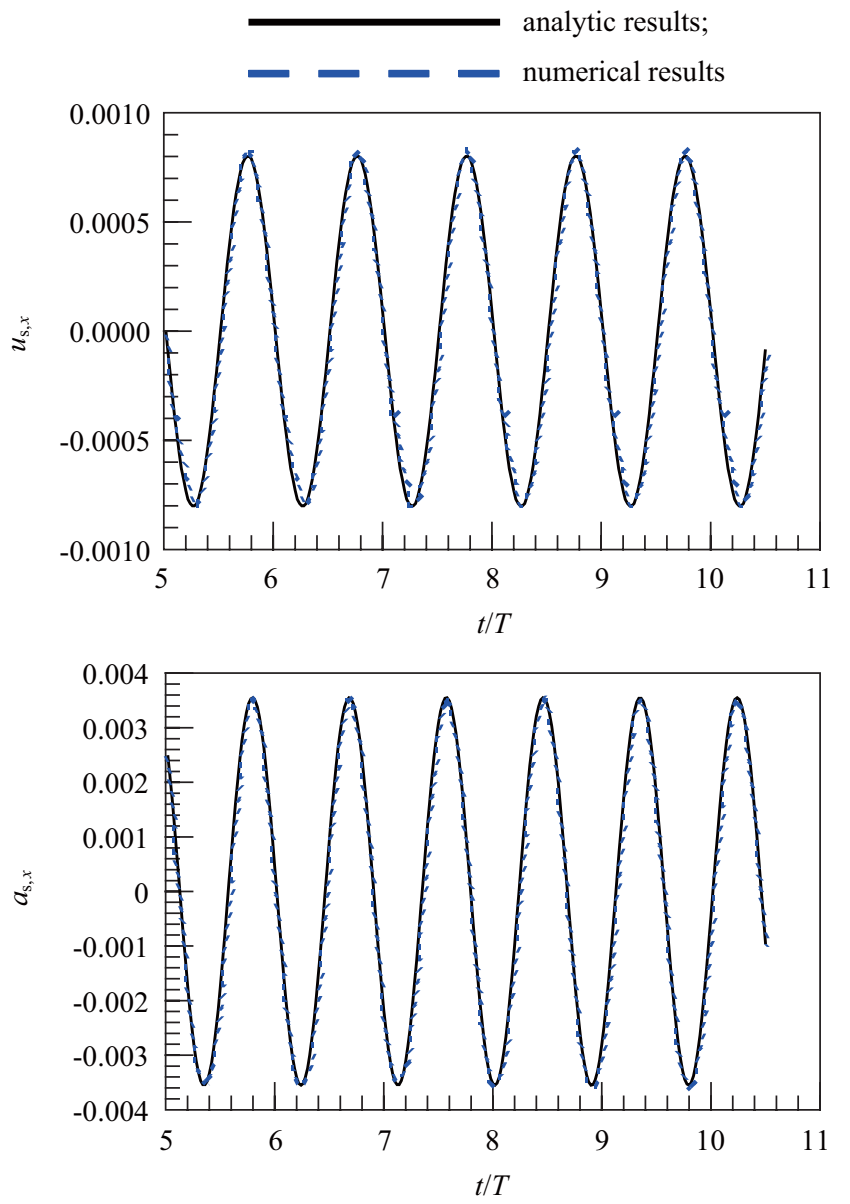

Fig. 5. Comparisons of the diffraction velocity and acceleration between numerical results and theory at a point $(0.8,0.0,-3.0)$.

( 8 (height of the cylinder $)+2$ (radius of the cylinder $))$ ) elements on the body surface, and 448 (16 (circumferential) $\times(16$ (radius of the still water surface) +12 (radius of the damping area))) elements on the water surface.

The incident regular wave with amplitude $A / a=0.1$ and wave number $k a=2.0$ is considered in the following case. Figure 5 shows the comparisons of $u_{\mathrm{s}, x}$ and $a_{\mathrm{s}, x}$ at point $(0.8$, $0.0,-3.0)$ (obtained by (13) and (14)) and analytic solutions [13]. From the figures it can be seen that $u_{\mathrm{s}, x}$ and $a_{\mathrm{s}, x}$ are in accord with analytic solutions very well, which indicates that the solved $u_{\mathrm{s}, x}$ and $a_{\mathrm{s}, x}$ are correct.

With the water-particle velocities and accelerations of diffraction waves and incident waves, substituted into Morison formula, the wave loads on small-scale piles can be solved.

Firstly, with different upper cylinder radius, the influence of diffraction field on the wave load on small-scale piles was investigated. It is assumed that the pile diameter $D=2 b=0.5 a$, which keep unchanged, and the cylinder diameter is $D_{0}$, which is different times of $a$; the value of $D_{0} / D$ was used as the reference for comparison.

Figure 6 shows the comparison of forces on pile from incident waves and diffraction waves separately at different val- 


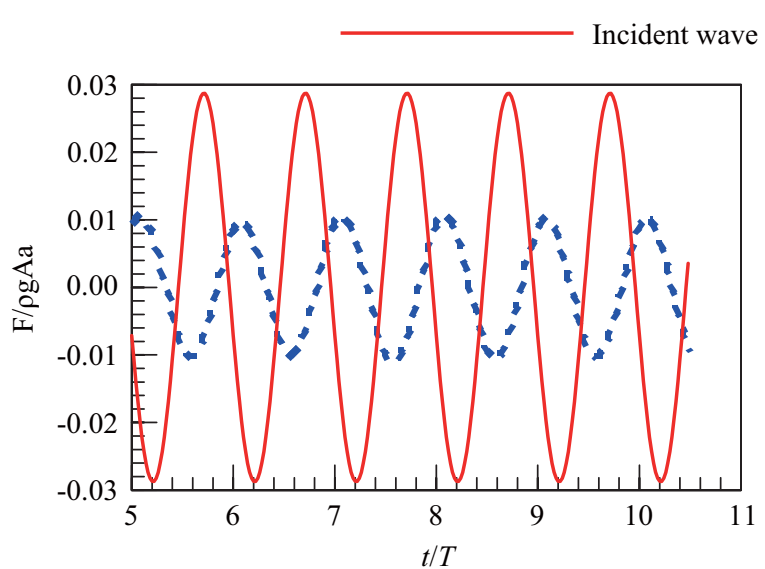

(a)

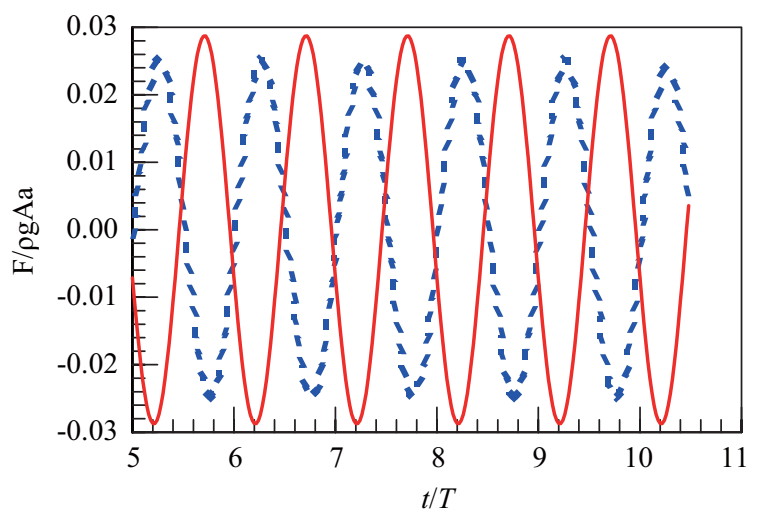

(c)

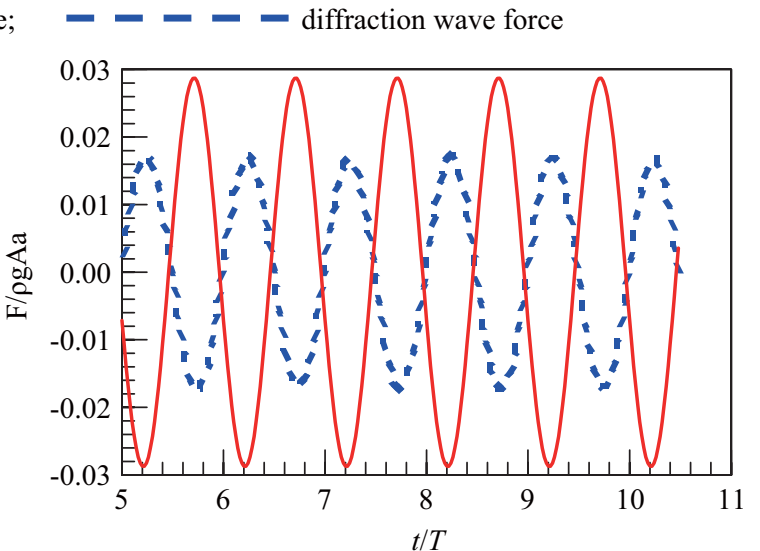

(b)

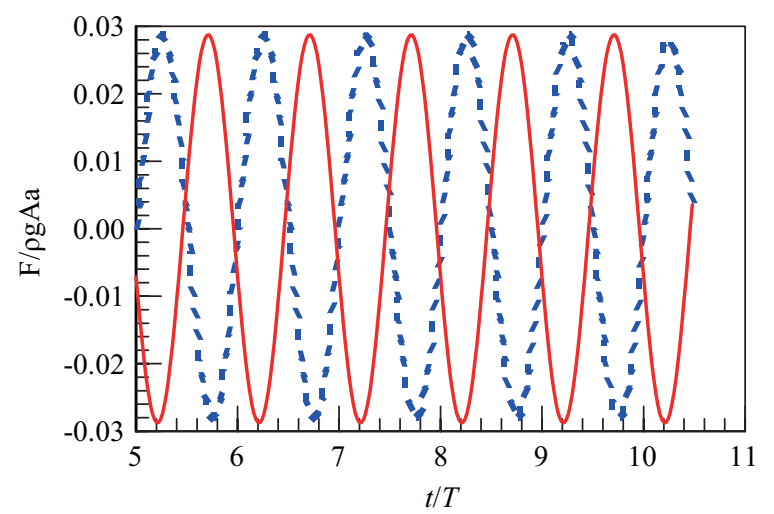

(d)

Fig. 6. Comparison of incident and diffraction wave forces on the pile at $D_{0} / D=2.0,4.0,6.0,8.0$.

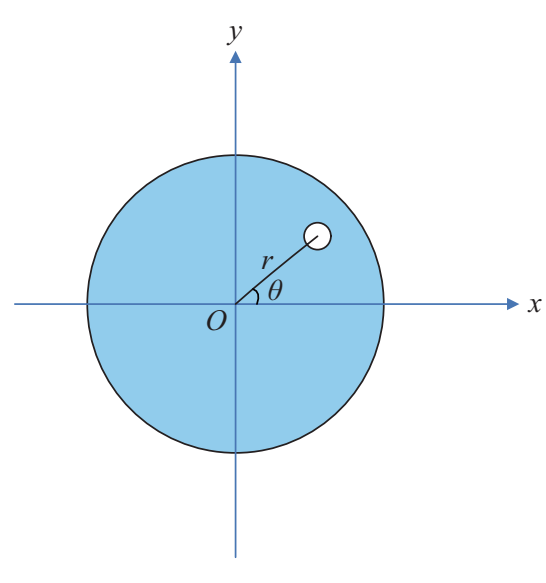

Fig. 7. Projection of the piles at Oxy plane.

ues of $D_{0} / D$. Because the incident wave field is fixed, the incident-wave forces in Fig. 6 are the same, i.e., with the amplitudes of $2.87 \times 10^{-2}$. Figure 6(a) shows the comparison at $D_{0} / D=2.0$, from which it can be seen that the amplitude of diffraction-wave force is $1.02 \times 10^{-2}$; Fig. 6(b) shows the comparison at $D_{0} / D=4.0$, in which the amplitude of diffraction- wave force is $1.68 \times 10^{-2}$, and the phase position turns gradually opposite to the incident wave force; Fig. 6(c) shows the comparison at $D_{0} / D=6.0$, where the amplitude of diffraction-wave force is $2.54 \times 10^{-2}$; Fig. $6(\mathrm{~d})$ shows the comparison at $D_{0} / D=$ 8.0, in which the amplitude of diffraction-wave force is $2.86 \times$ $10^{-2}$. From the figures it can be seen that with the increased radius of the upper large-scale cylinder, the produced diffraction field became intensified gradually, which was followed by increased diffraction-wave force acted on small-scale piles. Due to the selected position of the chosen pile, the phases of diffraction-wave force and incident-wave force had certain difference.

For the second case, the influence of diffraction field on the wave load on small-scale piles at different positions was investigated. For the convenience of illustration, the coordinates of the pile's cross section central is denoted by polar coordinates $(r, \theta)$ which just coincides with the $O x y$ plane, as shown in Fig. 7.

The incidence condition remained unchanged in calculation. The radius of the large-scale cylinder is $a$, while its other sizes are the same as in the first case. According to the symmetry principle, only the wave loads on the piles within $\theta \in\left[0^{\circ}, 180^{\circ}\right]$ was investigated.

Figure 8 shows the comparison of incident-wave force and 


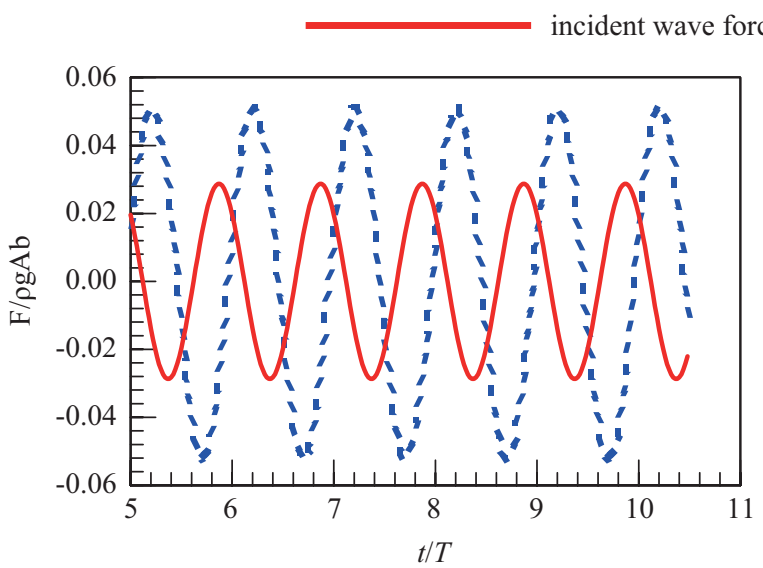

(a)

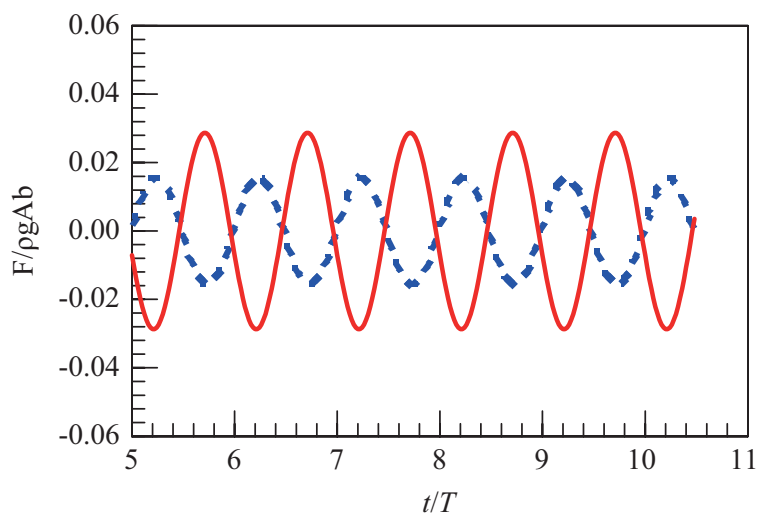

(c)

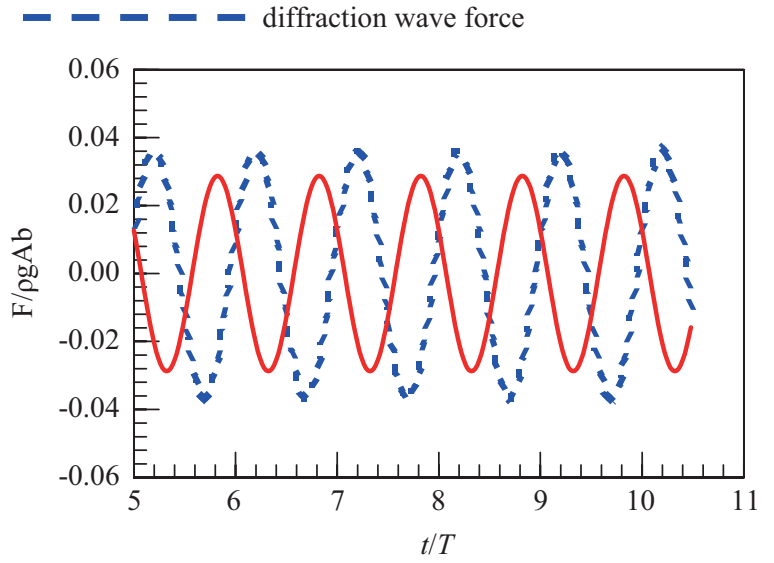

(b)

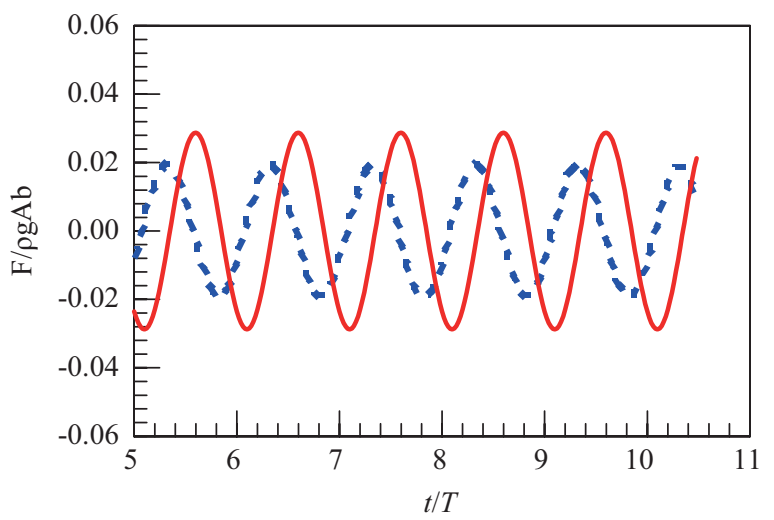

(d)

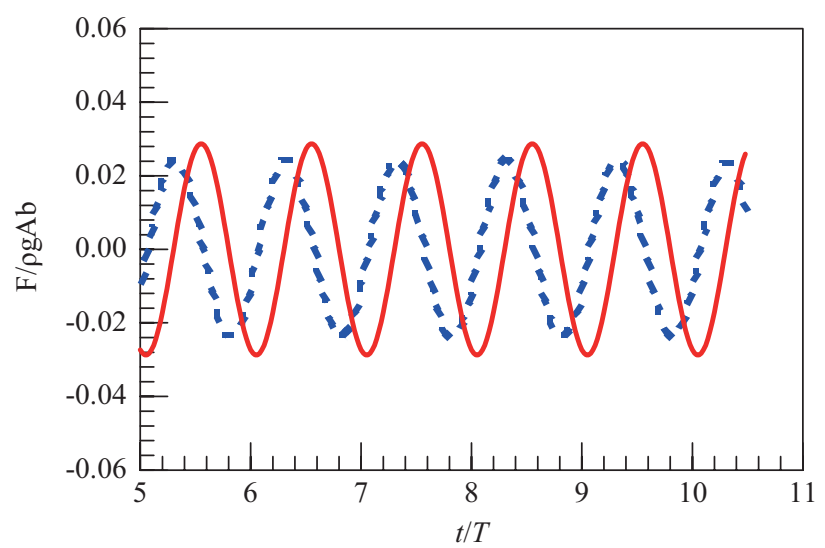

(e)

Fig. 8. Comparison of incident and diffraction wave forces on the piles at $r=0.5 a, \theta=0^{\circ}, 4^{\circ}, 90^{\circ}, 135^{\circ}, 1^{\circ}$.

diffraction-wave force on poles at $r=0.5 a$ and $\theta=0^{\circ}, 45^{\circ}, 90^{\circ}$, $135^{\circ}$ and $180^{\circ}$. Same with those in Fig. 6, the amplitudes of incident-wave forces are all the same due to unchanged incident wave field, i.e., $2.87 \times 10^{-2}$, but their phases changed with different pile positions. Figure 8(a) shows the comparison at $\theta=0^{\circ}$, from which it can be seen that the amplitude of diffraction wave force is $5.08 \times 10^{-2}$, being 1.77 times of the incidentwave force; Fig. 8 (b) shows the comparison at $\theta=45^{\circ}$, in which the amplitude of diffraction wave force is $3.64 \times 10^{-2}$, being
1.27 times of diffraction wave force; Fig. 8(c) shows the comparison at $\theta=90^{\circ}$, in which the amplitude of diffraction wave force is $1.57 \times 10^{-2}$, being $54.8 \%$ of diffraction wave force; Fig. 8(d) shows the comparison at $\theta=135^{\circ}$, in which the amplitude of diffraction wave force is $1.95 \times 10^{-2}$, being $68.1 \%$ of diffraction wave force; Fig. 8(e) shows the comparison at $\theta=180^{\circ}$, in which the amplitude of diffraction wave force is $2.46 \times 10^{-2}$, being $85.7 \%$ of diffraction wave force.

Figure 9 shows the amplitude comparison of incident, dif- 

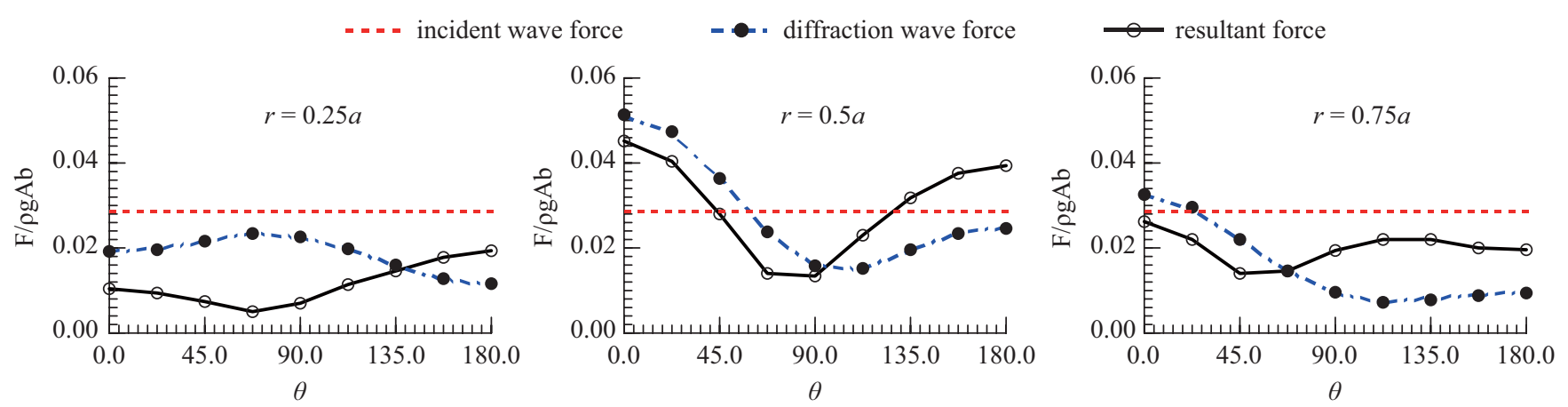

Fig. 9. Comparison of incident, diffraction and total wave forces with different $\theta$.

fraction and the resultant forces at $r=0.25 a, 0.5 a, 0.75 a$ and different $\theta$, from which it can be seen that the amplitude of incident wave force is a constant, with the value of $2.87 \times 10^{-2}$.

For $r=0.25 a$ and $0.75 a$, the resultant force is smaller than the incident wave force, and it is safe to design according to incident wave force.

For $r=0.5 a$, for diffraction wave force, the amplitude is greater than the incident wave force within $\theta \in\left[0^{\circ}, 60^{\circ}\right]$, and the amplitude is $5.07 \times 10^{-2}$ at $\theta=0^{\circ}$, reaching the maximum; when $\theta>60^{\circ}$, the diffraction wave force is smaller than the incident wave force; around $\theta=115^{\circ}$, the amplitude reaches the minimum, i.e. $1.52 \times 10^{-2}$.

For the resultant force, the amplitudes at the back side of the cylinder within approximate $\theta \in\left[0^{\circ}, 45^{\circ}\right]$ and at the wave side within $\theta \in\left[125^{\circ}, 180^{\circ}\right]$ are both greater than incident wave force; moreover, the amplitude reaches the maximum at $\theta=0^{\circ}$ with the amplitude of $4.52 \times 10^{-2}$, which is 1.57 times of the incident wave force. Under this condition, the diffractioninduced wave load on piles should be completely considered.

\section{CONCLUSIONS}

By taking wave diffraction from a fixed composite object as the example, the wave force on small-scale piles was calculated in diffraction filed, and the conclusions include:

1. For the given incident conditions in this paper, with the increasing of the ratio between large and small-scale objects diameters, the diffraction-wave force acted on small-scale piles became intensified gradually, which was followed by produced diffraction field increased. When $D_{0} / D=8.0$, the amplitude of diffraction-wave force is $2.86 \times 10^{-2}$, which is approximately equal the amplitude of incident-wave force $\left(2.87 \times 10^{-2}\right)$. Due to the selected position of the chosen pile, the resultant force may be smaller than incident-wave force because the phase difference between incident-wave force and diffraction-wave force.

2. The diffraction-wave force on the piles is sharp difference with different positions in the diffraction filed, and it also brings about differences on the resultant force.
For the resultant force, the amplitude reaches the maximum at $\theta=0^{\circ}$ with the amplitude of $4.52 \times 10^{-2}$, which is 1.57 times of the incident wave force. Under this condition, the diffraction-induced wave load on piles should be considered fully.

From the above studies, for a complicated structure composed of large-scale cylinders and small-scale members, the influence of diffraction or radiation field on the small-scale members or piles is remarkable. And the diffraction or radiation wave loads on piles should be considered completely in engineering design.

\section{ACKNOWLEDGMENTS}

The authors gratefully acknowledge the financial support provided by the National Science and Technology Major Project of China (Grant No. 2008ZX05026-02-02); Natural Science Foundation of China (Grant No. 10772040, 50709005) and Central Commonweal Research Institute Basic R\&D Special Foundation of TIWTE (Grant No. TKS090201).

\section{REFERCNCES}

1. Bai, W. and Eatock Taylor, R., "Fully nonlinear simulation of wave interaction with fixed and floating flared structures," Ocean Engineering, Vol. 36, pp. 223-236 (2009).

2. Bai, W. and Eatock Taylor, R., "Higher-order boundary element simulation of fully nonlinear wave radiation by oscillating vertical cylinders," Applied Ocean Research, Vol. 28, pp. 247-265 (2006).

3. Chakrabarti, S. K., Hydrodynamics of Offshore Structures, Computational Mechanics Publications, London, Great Britain (1987).

4. Chakrabarti, S. K., Barnett, J., Kanchi, H., Mehta, A., and Yim, J., "Design analysis of a truss pontoon semi-submersible concept in deep water," Ocean Engineering, Vol. 34, pp. 621-629 (2007).

5. Chen, J. T. and Hong, H. K., Boundary Element Method, 2nd Ed., New World Press, Taipei, Taiwan (1992).

6. Chen, P. Y., Xue, H. X., Tang, W. Y., and Zhang, S. K., "Fatigue analysis of tubular joints in a truss spar platform," The Ocean Engineering, Vol. 25, pp. 15-20 (2007).

7. Ferrant, P., "Three-dimensional unsteady wave-body interactions by a Rankine boundary element method," Ship Technology Research, Vol. 40, pp. 165-175 (1993).

8. Fish, P. R., Dean, R. B., and Heaf, N. J., "Fluid-structure interaction in Morison's equation for the design of offshore structures," Engineering Structures, Vol. 2, pp. 15-26 (1980).

9. Isaacson, M. and Cheung, K. F., "Time domain second order wave dif- 
fraction in three dimension," Journal of Waterway, Port, Coastal and Ocean Engineering, ASCE, Vol. 118, pp. 496-516 (1992).

10. Isaacson, M. and Cheung, K. F., "Second order wave diffraction around two dimensional bodies by time domain method," Applied Ocean Research, Vol. 13, pp. 175-186 (1991).

11. Kim, M. H., Ran, Z., and Zheng, W., "Hull/mooring coupled dynamic analysis of a truss spar in time domain," Proceedings of Eleventh International Offshore and Polar Engineering Conference, Vol. 11, pp. 42-54 (2001).

12. Kurian, V. J., Montasir, A. A., and Narayanan, S. P., "Numerical and model test results for truss spar platform," Proceedings of Nineteenth International Offshore and Polar Engineering Conference, Vol. 11, pp. 99-104 (2009).

13. Li, Y. C. and Teng, B., Wave Action on Maritime Structures, 2nd Ed.,
Ocean Press, Peking, China, pp. 56-60 (2002).

14. Luo, Y. H., Lu, R., Wang, J., and Berg, S., "Time-domain fatigue analysis for critical connections of truss spar," Proceedings of Eleventh International Offshore and Polar Engineering Conference, Vol. 1, pp. 362-368 (2001).

15. Morison, J. R., O’Brien, M. P., Johnson, J. W., and Schaaf, S. A., "The force exerted by surface wave on piles," Petroleum Transactions, AIME 18, pp. 149-157 (1950).

16. Teng, B. and Eatock Taylor, R., "New high-order boundary element methods for wave diffraction/radiation," Applied Ocean Research, Vol. 17, pp. 71-77 (1995).

17. Zhang, F., Yang, J. M., and Li, R. P., "Numerical research on hydrodynamics of a new cell-truss Spar platform," The Ocean Engineering, Vol. 25, pp. 1-8 (2007). 